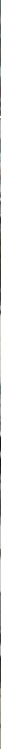

\title{
Wer ist der Souverän?
}

Liebe Kolleginnen und Kollegen,

Alle vier Jahre wählen wir einen neuen Bundestag. Nach dieser Urwahl ist die Wahl des Bundeskanzlers und damit der neuen Regierung eine der ersten und wichtigsten Aufgaben des neu gewählten Parlaments. Die neue Mehrheit im Parlament wählt eine neue Regierung für die folgende Legislaturperiode, selbstverständlich - oder doch nicht?

Mit dem Gesetz zur Modernisierung der gesetzlichen Krankenversicherung wurde 2005 die Hauptamtlichkeit für die Vorstände der Kassenärztlichen und Kassenzahnärztlichen Vereinigungen eingeführt. Angeblich, um durch eine „Professionalisierung" Waffengleichheit mit den Krankenkassen herzustellen. Zugleich wurde die Amtszeit auf sechs Jahre festgelegt. Nach inzwischen fast elf Jahren befinden sich die hauptamtlichen Vorstände der KZVen nun kurz vor dem Ende ihrer zweiten Amtsperiode. Ende 2016 finden Neuwahlen zu den KZVVertreterversammlungen (VV) statt. Eine der wenigen Möglichkeiten für die Vertragszahnärzte, Einfluss zu nehmen und gesundheitspolitisch Weichen zu stellen - oder doch nicht?

\section{Vorgezogene KZV-Vorstandswahlen}

Bereits vor dem Ende der ersten Amtsperiode wurde der Vorstand der KZV Nordrhein von der VV für die nächsten sechs Jahre im Amt bestätigt. Den Wählern wurde also faktisch die Möglichkeit genommen, Einfluss auf die Führung der KZV zu nehmen. Vor den nun anstehenden Wahlen wird in verschiedenen KZV-Bereichen darüber diskutiert, dem Beispiel zu folgen und die Vorstände für die Amtsperiode 2017 bis 2022 bereits vor der anstehenden Neuwahl von den jetzigen Vertreterversammlungen wählen zu lassen. In diesem Fall würde eine gewählte VV den Vorstand nicht nur für eine Legislaturperiode von sechs Jahren bestimmen, sondern für zwei Legislaturperioden, also volle zwölf Jahre. Die Urwahl zur Vertreterversammlung wäre für die Wahl des Vorstandes ohne Bedeutung. Erst am Ende der Amtsperiode könnte eine mögliche neue Mehrheit die Vorstandswahl für die folgende Amtsperiode beeinflussen.

Befürworter einer vorgezogenen Vorstandswahl möchten die Kontinuität der Vorstandsarbeit gesichert sehen. Außerdem bräuchten die Kandidaten für die Vorstandswahl Planungssicherheit. Die Handlungsfähigkeit der KZV sei nicht anders zu gewährleisten.

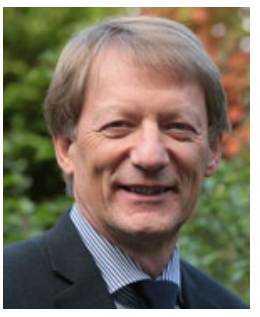

Dr. Peter Bührens

Stellvertretender FVDZ-Bundesvorsitzender

Inzwischen sollen weitere vier KZVen dem Beispiel von Nordrhein gefolgt sein und ihre Satzungen geändert haben, um eine vorgezogene Vorstandswahl zu ermöglichen.

\section{Bärendienst für die Selbstverwaltung}

Einige Aufsichtsbehörden haben diese Satzungsänderungen bereits genehmigt, in anderen Bundesländern wurden entsprechende Regelungen als unzulässig abgelehnt. Möglicherweise werden sich die zuständigen Gerichte mit dieser Frage beschäftigen müssen.

KZVen, die diese Satzungsänderungen betreiben, erweisen damit der Selbstverwaltung und der Kollegenschaft einen Bärendienst. Man könnte auch sagen: Sie machen sich zum Vollstrecker einer Professionalisierung im Sinne von Ulla Schmidt. Was ist das für ein Selbstverständnis von Selbstverwaltung? Und welche Wirkung geht von einem solchen Signal auf unseren standespolitischen Nachwuchs aus?

Die uns von Ulla Schmidt per Gesetz verordnete Hauptamtlichkeit mitsamt der vorgeschrieben sechsjährigen Amtsdauer wurde von der Hauptversammlung des Freien Verbandes in Warnemünde und von der KZBV-Vertreterversammlung in München abgelehnt. Ich teile nicht die Auffassung, dass sie sich zwischenzeitlich als Segen für die Kollegenschaft erwiesen hat.

Ich wünsche Ihnen im Namen des gesamten FVDZ-Bundesvorstandes ein frohes Weihnachtsfest und einen guten Rutsch ins Jahr 2016!

Ihr

$$
\text { Pulsuns }
$$

Dr. Peter Bührens 